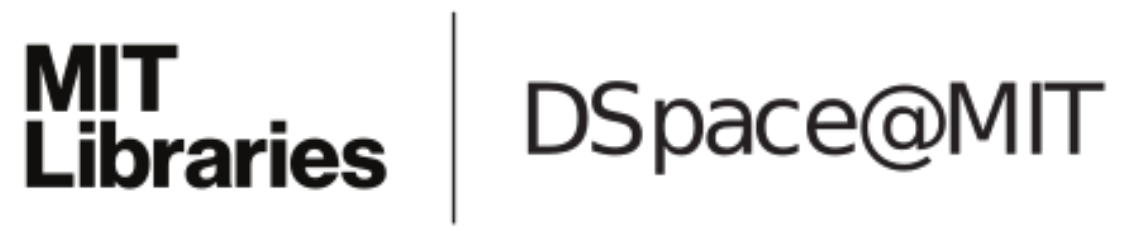

\author{
MIT Open Access Articles
}

Impact of the cracks lost in the imaging process on computing linear elastic properties from 3D microtomographic images of Berea sandstone

The MIT Faculty has made this article openly available. Please share how this access benefits you. Your story matters.

Citation: Zhang, Yang, and M. Nafi Tokso\#z. "Impact of the Cracks Lost in the Imaging Process on Computing Linear Elastic Properties from 3D Microtomographic Images of Berea Sandstone." Geophysics 77.2 (2012): R95. (02012 Society of Exploration Geophysicists

As Published: http://dx.doi.org/10.1190/geo2011-0126.1

Publisher: Society of Exploration Geophysicists

Persistent URL: http://hdl.handle.net/1721.1/73617

Version: Final published version: final published article, as it appeared in a journal, conference proceedings, or other formally published context

Terms of Use: Article is made available in accordance with the publisher's policy and may be subject to US copyright law. Please refer to the publisher's site for terms of use. 


\title{
Impact of the cracks lost in the imaging process on computing linear elastic properties from 3D microtomographic images of Berea sandstone
}

\author{
Yang Zhang ${ }^{1}$ and M. Nafi Toksöz ${ }^{2}$
}

\begin{abstract}
With the current developments in imaging/computational techniques and resources, computational rock physics has been emerging as a new field of study. Properties of rocks are examined by carrying out extensive numerical simulations on rocks that have been digitized using high-resolution X-ray CT scans. The ultimate goal of computational rock physics is to supplement the traditional laboratory measurements, which are time consuming, with faster numerical simulations that allow the parameter space to be explored more thoroughly. We applied the finite-element method to compute the static effective elastic properties from 3D microtomographic images of Berea sandstone saturated with different fluids. From the computations, we found discrepancies between the numerical results and the laboratory measurements. The reason for such a problem is the loss of small features, such as fine cracks and micropores, in the digitized matrix during the imaging and aggregation
\end{abstract}

process. We used a hybrid approach, combining the numerical computation and the effective media theories - the differential effective medium model and the Kuster-Toksöz model — to deduce the lost cracks by a very fast simulated annealing method. We analyzed the sensitivity of the inverted results — the distributions of crack aspect ratios and concentrations - to the clay content. We found that the inverted crack distribution is not so sensitive to clay content. Compared with the effect of cracks on the computed effective elastic properties, clay has only a secondary effect. Our approach can recover the lost cracks and is capable of predicting the effective elastic properties of the rocks from the microtomographic images for different fluid saturations. Compared with the traditional inversion schemes, based only on the effective media theories, this hybrid scheme has the advantage of utilizing the complex microstructures that are resolved in the imaging process, and it helps define the inversion space for crack distribution.

\section{INTRODUCTION}

In seismic data interpretation, understanding the relationships between elastic properties of rocks, pore spaces and fluids are critical and form the basis for reservoir characterization and monitoring. Effective properties of porous rocks depend highly on pore spaces, solid phases, and interactions between these two. To accurately predict the properties of rocks requires precise information on their complex microstructures and the ability to computationally solve the large 3D problems.

Traditionally, researchers formulate empirical relationships from laboratory experiments to help predict properties of rocks (Wyllie et al., 1956, 1958; Han, 1986). However, such relationships are too simple to be predictive for a wide range of rocks and lack the detailed description of the microstructures of rocks. The emergence of computational rock physics fills this gap and expands the research on rock physics in a new direction (Roberts and Gorboczi, 2000; Arns et al., 2002; Saenger et al., 2004; Grechka and Kachanov, 2006; Saenger, 2008).

With current advanced imaging techniques, such as microcomputed tomography $(\mu$-CT), micro X-ray CT (Flannery et al., 1987; Spanne et al., 1994), focused ion beam scanning electron microscopy (FIBSEM), laser confocal microscopy (Fredrich et al., 1995), and magnetic resonance imaging (MRI), we now are able to generate $3 \mathrm{D}$ images of rocks in high resolution. The usual voxel resolution is about $1-10 \mu \mathrm{m}$ or higher. Such high-resolution images

Manuscript received by the Editor 30 March 2011; revised manuscript received 20 November 2011; published online 2 March 2012.

${ }^{1}$ Formerly Massachusetts Institute of Technology, Department of Earth, Atmospheric, and Planetary Sciences, Earth Resources Laboratory, Cambridge, Massachusetts, USA; Presently GeoReservoir Research, Inc., Stafford, Texas, USA. E-mail: yzhang@georeservoir.com.

${ }^{2}$ Massachusetts Institute of Technology, Department of Earth, Atmospheric, and Planetary Sciences, Earth Resources Laboratory, Cambridge, Massachusetts, USA. E-mail: toksoz@mit.edu.

(C) 2012 Society of Exploration Geophysicists. All rights reserved. 
provide direct measurements of the complex morphology of porous rocks. Combining these with computational techniques, we can calculate the material properties such as diffusivity, elasticity, permeability, and conductivity. The ultimate goal of the development of these computational simulations and methods is to supplement physical experiments, which have traditionally been carried out in laboratories and are time consuming, with faster numerical simulations that allow the parameter space to be explored more thoroughly.

However, the X-ray CT imaging technique is unable to obtain images of rocks with high enough resolution to illuminate every crack or micropore between and within grains for sandstone or carbonates. Even if it were able to do so, current computational resources limit our ability to solve such huge 3D problems on a realistic scale because the resolution required would be down to nanometer scale. In general, the loss of small features of rocks due to the imaging process not only affects the computed effective elastic properties (i.e., Arns et al., 2002) but also the computed effective permeability and conductivity (i.e., Zhan et al., 2010).

Arns et al. (2002) carried out extensive numerical computations on 3D digitized rock samples of Fontainebleau sandstone with variable porosities and compared their numerical predictions to those from Gassmann's model (Gassmann, 1951) and experimental measurements. The numerical predictions, using the finite-element method (FEM), for Fontainebleau sandstone generally matched those predicted from Gassmann's equations and experimental measurements. However, there were some discrepancies that (1) the computed velocities for both dry and water/oil-saturated cases were generally higher than experimental measurements - this was more obvious for shear moduli; and (2) computed velocities of P-waves for dry rocks were higher than those for water/oil-saturated ones, which contradicted laboratory observations for sandstone. We also found the same contradictions in our own computations on digitized 3D Berea sandstone. One explanation Arns (2002) gave for such discrepancies was that the periodic boundary condition was used in the FEM solver. However, we think that this is not the real reason behind these observations. We believe that this issue relates to an
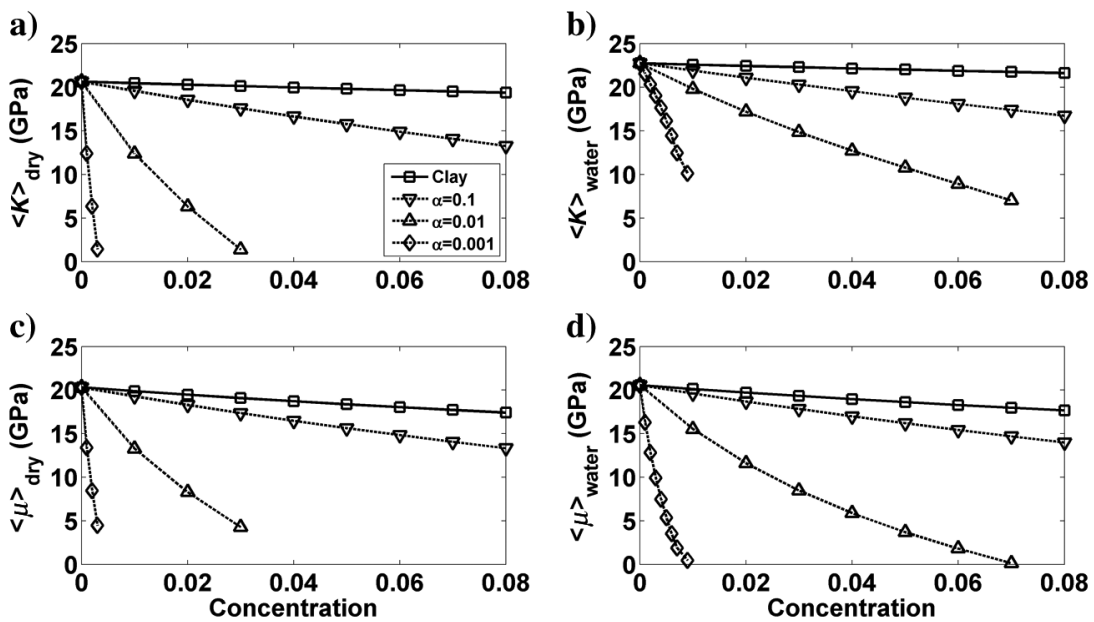

Figure 1. The variations of the computed effective elastic moduli $\langle K\rangle$ and $\langle\mu\rangle$ for dry and water-saturated digitized rock samples with the concentration of clay and cracks, respectively. Cracks considered have aspect ratios: 0.1, 0.01, and 0.001. (a) Bulk moduli $\langle K\rangle$ and (c) shear moduli $\langle\mu\rangle$ for dry rock samples; (b) bulk moduli $\langle K\rangle$ and (d) shear moduli $\langle\mu\rangle$ for water-saturated samples. assumption we make about the solid phase properties in numerical computation. We assume that the solid phase is made up of a continuum of quartz. As will be pointed out in this paper, cracks and other small features of rocks, unresolved during the imaging process, actually make the rock matrix weaker. Ignoring the effects of cracks gives rise to the overpredicted velocities of well-cemented sandstone.

Zhan et al. (2010) computed the effective permeability and conductivity of the same digitized Berea sandstone as the ones we are using in this paper. In the resolution tests, they found that by lowering the resolution of the digitized images according to the majority rule, the computed effective permeabilities decreased due to the loss of the small channels that originally existed and also contributed to fluid flow at higher resolution.

In the current practice of computational rock physics, the contributions of the small features lost in the imaging process to the final computed effective properties of digitized rocks often are not acknowledged. In this paper, we will address this issue by carrying out extensive computations on digitized Berea sandstone and propose a hybrid method that combines the numerical computation with the theoretical models, to recover the cracks lost in the imaging process. Compared with usually "pure" Fontainebleau sandstone, Berea sandstone contains small amounts of clay, which makes this problem even more complicated. However, given the same amount of volume fraction, the impact of small cracks, especially those with smaller aspect ratios, is much more significant than that of clay on the computed effective elastic properties as shown in Figure 1. We will give a detailed description of how we obtain the data shown in the Figure 1 in the following section of this paper. Due to the first order impact of cracks on the computed effective elastic properties, we will focus mostly on recovering the crack distribution by using a very fast simulated annealing method (VFSA) and a limited number of laboratory measurements. As for the clay, though it causes a secondary effect, we will still study its effect by analyzing the sensitivity of the inverted crack distribution to the clay content.

The VFSA inversion works directly on the numerically predicted effective elastic moduli of the digitized 3D Berea sandstone for dry and water-saturated cases that only include the pore space resolved in the imaging process. By using these effective elastic properties in the inversion, we capture the effect of complex microstructures of pore space resolved, and we are actually able to reduce the inversion space and solely target the inversion for small cracks lost. Finally, we will compute the effective elastic properties of rocks, including both the pore space resolved in the imaging process and the small cracks from the inversion, and we will compare these numerical predictions to laboratory data measured independently by different researchers.

\section{DIGITIZED 3D BEREA SANDSTONE FOR COMPUTATION}

\section{D X-ray CT images}

3D X-ray CT images for a cylindrical plug of Berea sandstone 500 were obtained from the Australia National University Digital Core Laboratory Consortium. The images have a total size of $1840 \times 1840 \times 1940$ pixels with the size 
of $2.8 \mu \mathrm{m}$ per pixel. This Berea sandstone contains low-density pore inclusions such as clay, feldspars, and microporosity that cause a transition in the intermediate portion of the attenuation histogram of X-ray CT measurements between two main peaks. Therefore, gray scale images from X-ray $\mathrm{CT}$ have been thresholded into two main phases: solid grain and pore space, corresponding to those two peaks, respectively. Clay is not distinguished from quartz as a third phase in the solid grain due to the imaging resolution. As an example, in Figure 2 we show the gray scale CT images of a cubic subset with the size of $250 \times 250 \times 250$ pixels that we extracted from the center of the original cylindrical plug. In Figure 3, we show the corresponding segmented images for this same subset, in which grains are represented in light gray and pores are in dark gray. More detailed description of this Berea sandstone can be found in the paper by Zhan et al. (2010).

\section{Representative element volume}

To numerically and experimentally determine the effective elastic properties of porous rocks, the samples have to be large enough to be considered as the representative element volume (REV) so as to represent the equivalent homogeneous medium. In this study, we actually take the sample of the size of $250^{3}$ pixels shown in Figure 3 as the REV and compute the static effective properties of this sample with the finite-element method (FEM) from NIST (National Institute of Standards and Technology) (Garboczi and Day, 1995; Garboczi, 1998). FEM solves the weak form of the linear elastic equations and utilizes iterative solvers such as the conjugategradient method to find the solutions. In FEM, each pixel is taken to be a trilinear finite element and constant strain boundary conditions are applied. The effective elastic properties are obtained from average stresses and strains. Because the constant strain boundary conditions are applied to this cubic subset, an upper bound of the effective elastic properties might have been computed as pointed out by Saenger (2008) if the sample is not an REV. Therefore, it is necessary for us to prove that the sample of size of $250^{3}$ pixels is large enough to be considered as an REV in the computation.

First, from the whole digitized sample of the Berea sandstone, whose size is $1840 \times 1840 \times 1940$ pixels, we cut two cubic subsets

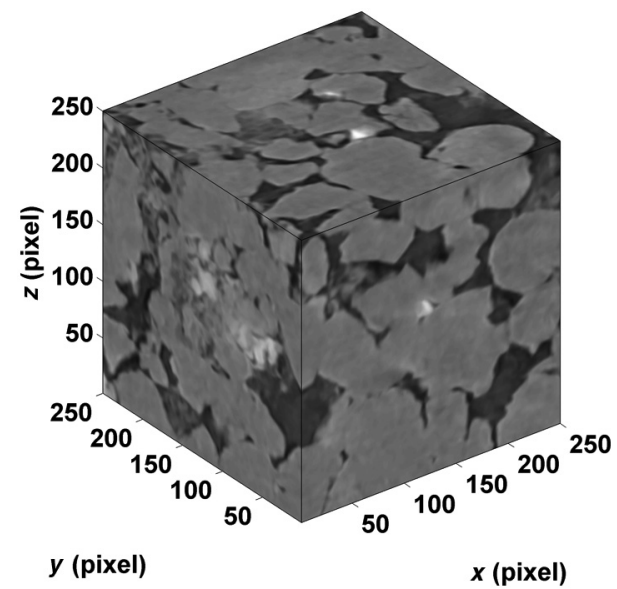

Figure 2. Gray scale X-ray CT images for the $250^{3}$ pixels cubic subset of a Berea sandstone. Dark gray represents the pore spaces. Gray matrix is mostly quartz. Different shades in the gray are due to clay and feldspar. with the sizes of $500^{3}$ and $750^{3}$, respectively. These two subsets center at the same point in the 3D space. Furthermore, we cut the $500^{3}$ into eight subsets with the size of $250^{3}$. In this way, we can have rock samples with different sizes spanning from $250^{3}$ to $750^{3}$ with porosities varying from $15.8 \%$ up to $25.3 \%$, correspondingly. We then compute the effective elastic moduli of dry and water-saturated cases for these samples at $40 \mathrm{MPa}$ pressure, assuming that the grains are occupied by pure quartz with bulk modulus $K=37 \mathrm{GPa}$, shear modulus $\mu=44 \mathrm{GPa}$, and mineral density $\rho=2650 \mathrm{~kg} / \mathrm{m}^{3}$ (Mavko et al., 1998); and inclusions in pores have $K_{\text {dry }}=0 \mathrm{GPa}, \mu_{\text {dry }}=0 \mathrm{GPa}$, and $\rho_{\text {dry }}=0 \mathrm{~kg} / \mathrm{m}^{3}$ for dry case, and $K_{\text {water }}=2.2 \mathrm{GPa}, \mu_{\text {water }}=0 \mathrm{GPa}$, and $\rho_{\text {water }}=1000 \mathrm{~kg} / \mathrm{m}^{3}$ for water-saturated case. In Figure 4, we show the effective bulk moduli $\langle K\rangle$ and shear moduli $\langle\mu\rangle$ computed for dry and water-saturated cases with samples at different sizes. Though porosity of different samples varies, especially those samples of size of $250^{3}$, their moduli vary with porosity linearly along the fitted dash line. Discussed by Arns et al. (2002) (Figure $4 \mathrm{a}$ and $4 \mathrm{~b}$ in their paper), a smaller sample can be considered as an REV for a sample at a specific porosity, if the distribution of the moduli computed from these smaller samples vary linearly with porosity, and the moduli from bigger samples follow the linear relationship. This means that though the porosity varies when smaller samples are selected, the smaller sample could be a representative one in terms of pore structures so as to give effective elastic properties in a linear relationship with each other. Therefore, the rock sample of size $250^{3}$ is large enough to capture the pore structures of the Berea sandstone so as to be considered as an REV at a given porosity.

Second, as pointed out by Saenger et al. (2006) and Saenger (2008), the lower and upper bounds of the effective elastic moduli observed with the static method become less significant when the dynamic simulation is applied. This means that if the moduli on a sample computed from a static method, such as the FEM, are close to or equal to those from a dynamic method, such as the finite difference method (FDM), the size of the sample should be large enough to be considered as an REV. As listed in Table 1, we compare the effective bulk $\langle K\rangle$ and shear $\langle\mu\rangle$ moduli for the dry and watersaturated digitized rock sample at size of $250^{3}$ with porosity of $19.2 \%$ computed from FEM and FDM, respectively. The moduli

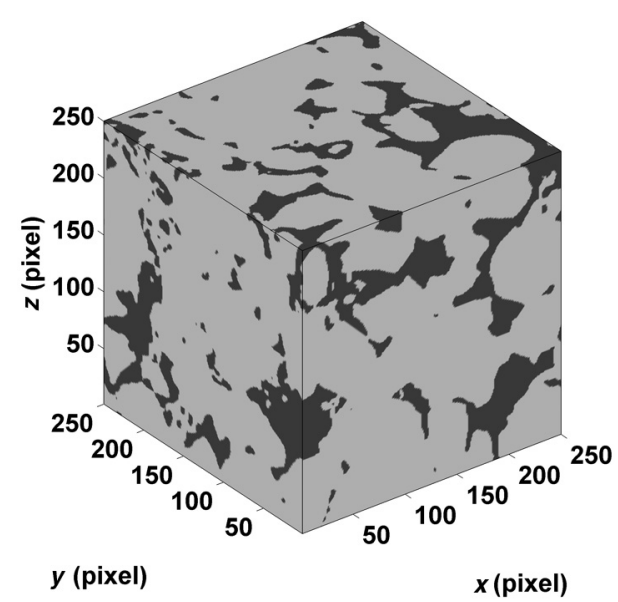

Figure 3. Segmented images for the $250^{3}$ pixels cubic subset of a Berea sandstone. Grains are represented in light gray and pores are in dark gray. 
of the solid grains have been chosen to be smaller than those of pure quartz, which allows a bigger time stepping in the FDM simulation and also takes into account the mineral compositions, such as clay and feldspars. The FEM is a static method from NIST, while the FDM is a dynamic method we have developed that computes the effective elastic moduli of a digitized rock sample by carrying out a stress-strain calculation (Zhang, 2010). From Table 1, we can see that the moduli computed from both of these two methods are quite close to each other, which means that the digitized rock sample of size of $250^{3}$ is an REV at the given porosity.

Third, as concluded by Arns et al. (2002), the representative image volume for well-cemented Fontainebleau sandstone should be a $120^{3}$ pixels cubic subset at resolution of $5.7 \mu \mathrm{m}$; this exactly corresponds to our sample with the size of $250^{3}$ pixels at $2.8 \mu \mathrm{m}$. Because in the digitized Berea sandstone only two phases - solid grain and pore space - are distinguished and no third phase for clay is included, from the computational point of view, the digitized Berea sandstone can be treated as a well-cemented sandstone. Therefore, we can choose such a cubic subset of the 3D Berea sandstone of size of $250^{3}$ as an REV in our computation, thanks to the similar static elastic responses of these two types of sandstone at the well-cemented condition.

\section{Laboratory data on Berea sandstone}

Berea sandstones with similar porosity and clay content demonstrate similar elastic properties. In our study we choose Berea sandstones with similar porosities (18.87\%-20.5\%) and clay content $(6 \%-9 \%)$ measured by different researchers. In Table 2, the P-wave velocity $\left(V_{\mathrm{P}}\right)$ and $\mathrm{S}$-wave velocity $\left(V_{\mathrm{S}}\right)$ for dry and water-saturated Berea sandstone measured by different researchers are listed. We can see that, though different Berea sandstone were measured in the laboratory, the maximum variations in velocities are around $2.6 \%$ for $V_{\mathrm{P}}$ and $3.5 \%$ for $V_{\mathrm{S}}$ for dry rocks, and $5.7 \%$ for $V_{\mathrm{P}}$ and $4.6 \%$ for $V_{\mathrm{S}}$ for water-saturated rocks. The differences due to variations in porosity and clay content are small among these Berea sandstones considering that different setups were used for laboratory measurements. This indicates that Berea sandstones with similar porosity and clay content do not vary much from one another in terms of pore structures and elastic properties. The laboratory data listed in Table 2 represent the properties of the Berea sandstone used in our calculations on the digitized Berea sample with similar porosity $(19.2 \%)$.

\section{ISSUES WITH THE EFFECTIVE ELASTIC PROPERTY PREDICTION WITHOUT CRACKS \\ Overprediction of the effective elastic property}
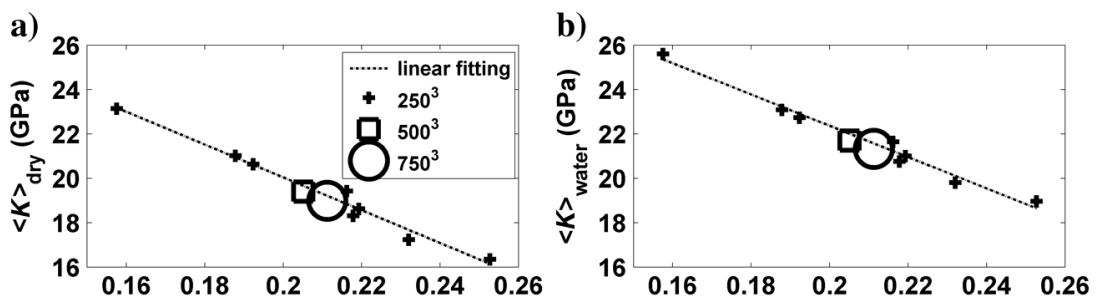

c)

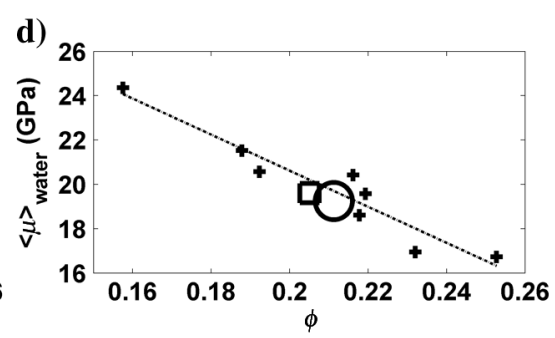

Figure 4. The computed effective elastic moduli $\langle K\rangle$ and $\langle\mu\rangle$ for dry and water-saturated digitized rock samples at different sizes, from $250^{3}$ to $750^{3}$ with different porosity $(\phi)$. (a) Bulk moduli $\langle K\rangle$ and (b) shear moduli $\langle\mu\rangle$ for dry rock samples; (c) bulk moduli $\langle K\rangle$ and (d) shear moduli $\langle\mu\rangle$ for water-saturated samples.

Table 1. Comparison of the effective bulk $\langle K\rangle$ and shear $\langle\mu\rangle$ moduli of the dry and water-saturated digitized rock sample of size of $250^{3}$ with porosity of $19.2 \%$ computed from the static method (FEM) and the dynamic method (FDM).

\begin{tabular}{llcc}
\hline & & $\langle K\rangle(\mathrm{GPa})$ & $\langle\mu\rangle(\mathrm{GPa})$ \\
\hline \multirow{2}{*}{ FEM } & Dry & 13.6 & 13.9 \\
& Water & 17.8 & 14.9 \\
\multirow{2}{*}{ FDM } & Dry & 13.5 & 14.1 \\
& Water & 17.7 & 15.0 \\
\hline
\end{tabular}

When discussing the REV of the rock samples in the previous section, we have carried out extensive computations for the effective elastic properties of dry and water-saturated rock samples at different sizes. In those computations we assume the grains are made up of a continuum of pure quartz and we model dry and watersaturated cases at high pressure (>40 MPa). The numerically predicted results for effective bulk $\langle K\rangle$ and shear $\langle\mu\rangle$ moduli and velocities of $\mathrm{P}$ - and S-waves for the rock sample of size of $250^{3}$ pixels with porosity $19.2 \%$ are listed in Table 3. These are compared with the laboratory data listed in the Table 2 (Winkler, 1985; Han, 1986; Best and McCann, 1995). Although the numerical predictions for dry and watersaturated cases satisfy Gassmann's equation, as Arns et al. (2002) demonstrated, we can see that our numerical predictions from FEM generally overestimate the effective elastic properties resulting in larger velocities. Also P-wave velocity predicted in the water-saturated case is less than that for the dry case; this usually occurs only for well-sintered glass-bead packs where there are no cracks.

\section{Crack loss in imaging process}

The reason for the overestimates of the numerical predictions is the loss of microstructures of rocks in X-ray CT scanning and the image segmentation afterward. As we know, small features like cracks or micropores in rocks are mostly in nanometer scale (Murphy et al., 1986). Although the X-ray CT technique can provide high-resolution images in micrometer scale, compared to small features in nanometer scale, its resolution is still too low to resolve 
the microstructures when we digitize rocks into CT images and separate phases afterward. Realizing these issues with the imaging process, Arns et al. (2007) introduced the concept of contact porosity to estimate the contact moduli between grains with effective medium theories, and Knackstedt et al. (2009) utilized SEM images, which have much higher resolution than CT images, to recover lost micropores. There is no unique way, so far, to resolve this issue related to the loss of microstructures in the imaging process. For samples like the Berea sandstone we used in our study, we are facing the same issues. Shown in Figure 5a is a 2D slice of an X-ray CT image cut from the 3D volume of the digitized Berea sandstone. The arrows in Figure 5a indicate cracks between grain contacts that are lost during the segmentation process. As shown in Figure 5b, after segmentation, we obtain a rock with continuous matrix where no microstructures between grains are resolved. As expected, the continuous matrix without cracks resulting from segmentation strengthens the rock frame and contributes to the overpredicted effective elastic properties of digitized rocks. Although there should have been other microstructures lost in the imaging process, we only focus on studying the effects of cracks because we demonstrate in Figure 1 that they have the most impact on the effective elastic properties of rocks, as discussed by many researchers (Walsh, 1965; Kuster and Toksöz, 1974; O'Connell and Budiansky, 1977; Hudson, 1980).

\section{INVERSION OF CRACK DISTRIBUTION WITH THE VERY FAST SIMULATED ANNEALING METHOD}

As discussed in the previous section, we believe that the loss of cracks during the imaging process is the main cause of higher predicted effective elastic properties of rocks. Therefore, we have to modify the continuous matrix of digitized rocks by taking the cracks into account. To accomplish this, we utilize a very fast simulated annealing method (VFSA) as the key inversion frame to recover the distribution of cracks lost in the imaging process. In such inversion, we incorporate a hybrid scheme combining the numerical computation with the theoretical models - the differential effective medium theory and the Kuster-Toksöz model (Kuster-Toksöz, 1974).

\section{Differential effective medium theory}

Note that instead of working directly on a continuous matrix to invert crack distribution, we first start with the numerically predicted effective moduli of the rock frame. For example, moduli for dry- and water-saturated cases are listed in Table 3, where no clay and crack are included in the solid grain of the digitized
Berea sandstone. Solid grain containing a small amount of clay can be treated in the same way, as we will discuss in detail in the following section. According to the differential effective medium theory (DEM), for a composite consisting of two phases, by choosing a preferred host material, we can incrementally add other phases or inclusions into the host. For the composite host medium at some porosity value $\phi$, the effective moduli $K^{*}(\phi+d \phi)$ and $\mu^{*}(\phi+d \phi)$ after a small portion of the composite host has been replaced by inclusions of the other phase, can be obtained by

$$
\begin{aligned}
& (1-\phi) \frac{d}{d \phi}\left[K^{*}(\phi)\right]=\left(K_{i}-K^{*}\right) P^{*}, \\
& (1-\phi) \frac{d}{d \phi}\left[\mu^{*}(\phi)\right]=\left(\mu_{i}-\mu^{*}\right) Q^{*}
\end{aligned}
$$

where $K^{*}(\phi)$ and $\mu^{*}(\phi)$ are the effective moduli to be estimated after adding a small portion of the second phase, $K_{i}$ and $\mu_{i}$ are moduli of the second phase, and $P^{*}$ and $Q^{*}$ are geometric factors that depend on the shapes of inclusions of the second phase (Berryman, 1992; Mavko et al., 1998).

For our case, first we treat the continuous solid containing any small amount of clay as the host that occupies the whole cubic domain without pores and cracks, and second, add the pore spaces resolved and cracks lost in the imaging process as the second phase into the host. There are two ways of adding pores and cracks into host according to the DEM: We can add pore spaces first, followed by cracks, or vice versa. In practice, the final effective elastic properties obtained from these two different ways of adding the second phase can be quite different for some cases. The numerical predictions, i.e., those listed in Table 3, can be taken as the intermediate effective properties of rocks after adding only pore spaces resolved in the imaging process. Therefore, starting with these results, we can invert distribution of cracks that should have existed in the continuous matrix by using the Kuster-Toksöz model (Kuster-Toksöz, 1974).

\section{The very fast simulated annealing inversion}

Simulated annealing (SA) is an optimization procedure that simulates thermodynamic annealing of melted solids. In an SA-based optimization method, an initial model is generated within predefined upper and lower bounds. A forward operator is applied to the model, and the predicted data are compared with observed data on the basis of a predefined cost function. The initial model is

Table 2. Laboratory measurements of velocities for dry and water-saturated Berea sandstone with similar porosities and clay content at high pressure (>40 MPa).

\begin{tabular}{lccccccc} 
& & & \multicolumn{3}{c}{ Dry } & \multicolumn{3}{c}{ Water } \\
\cline { 5 - 8 } & & Clay (\%) & Porosity $(\%)$ & $V_{\mathrm{P}}(\mathrm{m} / \mathrm{s})$ & $V_{\mathrm{S}}(\mathrm{m} / \mathrm{s})$ & $V_{\mathrm{P}}(\mathrm{m} / \mathrm{s})$ & $V_{\mathrm{S}}(\mathrm{m} / \mathrm{s})$ \\
\hline Han (1986) & BEREA 1 & 6 & 19.0 & 4040 & 2620 & 4150 & 2510 \\
Winkler (1985) & BEREA 2 & 9 & 18.9 & 3990 & 2630 & 4080 & 2540 \\
Best and McCann (1995) & BEREA 3 & 8 & 20.3 & 3963 & 2527 & & \\
\hline
\end{tabular}


perturbed and a new model is generated. The new model is unconditionally accepted if the cost function relative to the previous model is small. If the new model results in a higher cost function, the new model is still accepted with a certain probability:

$$
\Delta E=E_{k}-E_{k-1}
$$

If $\Delta E \leq 0$, then accept.

Otherwise $p=e^{-\Delta E / T}$ and $r=U[0,1]$

If $p \geq r$, then accept.

The SA method provides a finite probability to accept a generated model that has a higher cost function than does a previously accepted model. Thus, a finite probability exists to search in both the uphill and downhill directions, helping the algorithm to "jump out" of a local minimum.

Ingber (1989) proposed the VFSA, a new variant of SA that allows faster cooling. The VFSA algorithm generates new model parameters $m_{i}^{k+1}$ for model dimension $i$ and iteration $k+1$ from the corresponding model parameters $m_{i}^{k}$ at the previous iteration:

$$
m_{i}^{k+1}=m_{i}^{k}+y_{i}\left[\max \left(m_{i}\right)-\min \left(m_{i}\right)\right],
$$

where $\max \left(m_{i}\right)$ and $\min \left(m_{i}\right)$ are the predefined upper and lower bounds, respectively, of model parameters $m_{i}$; and $y_{i} \in[-1,1]$ is generated from a distribution function given by

$$
y_{i}=\operatorname{sgn}\left(r_{i}-\frac{1}{2}\right) T_{i}\left[\left(1+\frac{1}{T_{i}}\right)^{\left|2 r_{i}-1\right|}-1\right] \text {, }
$$

Table 3. Numerical predictions from finite-element simulation for the digitized Berea sandstone without cracks.

\begin{tabular}{lcccc} 
& $\langle K\rangle(\mathrm{GPa})$ & $\langle\mu\rangle(\mathrm{GPa})$ & $V_{\mathrm{P}}(\mathrm{m} / \mathrm{s})$ & $V_{\mathrm{S}}(\mathrm{m} / \mathrm{s})$ \\
\hline Dry & 20.6 & 20.3 & 4720 & 3080 \\
Water & 22.7 & 20.6 & 4637 & 2972 \\
\hline
\end{tabular}

where $r_{i}=U[0,1]$. When the generation of model parameters follows such a distribution, the global minimum is obtained using the model-parameter cooling schedule:

$$
T_{i}^{k}=T_{i}^{0} e^{-d_{i} k^{\beta}}
$$

where $T_{i}^{0}$ is the initial temperature for the parameters, $d_{i}$ is the temperature decay constant, and $\beta$ is the exponent constant controlling the cooling profile.

In the inversion for crack distribution, taking the saturated Berea sandstone in the intermediate stage of the DEM model as an isotropic and homogeneous elastic block and using the Kuster-Toksöz model, we can invert the distribution of spheroidal cracks with the VFSA inversion by fitting the laboratory measurements of velocities of P- and S-waves.

In the VFSA inversion, we invert for two sets of parameters, which are the aspect ratios $\left(\alpha_{n}\right)$ and concentrations $\left(c_{n}\right)$ of different sets of cracks, respectively. We assume the distribution of aspect ratios of cracks obeys a power function as suggested by Tran et al. (2008) as

$$
\alpha_{n}=10^{\frac{1-n}{2}} \text {. }
$$

Because most of the big pores have been resolved in the imaging process and we only focus on the small cracks lost, we choose $n=3, \ldots, 11$ to address these small cracks in the inversion.

The lower and upper bounds of the aspect ratios of cracks used for VFSA inversion are

$$
\left[\min \left(\alpha_{n}\right), \max \left(\alpha_{n}\right)\right]=\left[0.1 \alpha_{n}, 5 \alpha_{n}\right]
$$

Choosing the distribution of concentrations of cracks as

$c_{n}=\left[1 \times 10^{-2}, 1 \times 10^{-2}, 5 \times 10^{-3}, 5 \times 10^{-3}, \quad 5 \times 10^{-4}, 5 \times 10^{-4}, 5 \times 10^{-5}, 5 \times 10^{-5}, 5 \times 10^{-6}\right]$

to limit the total concentration of cracks to less than $\sim 3 \%$, we set the lower and upper bounds of the concentration of cracks to be

$\left[\min \left(c_{n}\right), \max \left(c_{n}\right)\right]=\left[0.01 c_{n}, c_{n}\right]$.
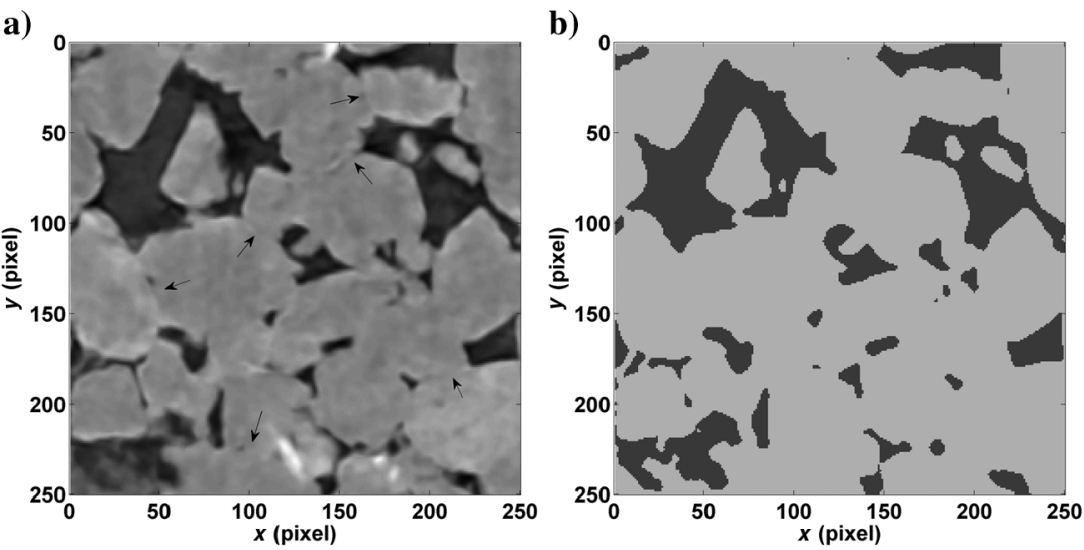

Figure 5. Two-dimensional slices of digitized Berea sandstone. (a) X-ray CT image in gray scale; (b) segmented image. We can clearly see the loss of cracks between grain contacts due to the imaging process, as indicated by arrows in (a).
In the VFSA inversion, we set $T_{i}^{0}=1, d_{i}=1$, and $\beta=0.3$. As a function of aspect ratio $\left(\alpha_{n}\right)$ and concentration $\left(c_{n}\right)$, the cost function measuring the difference between the numerical predicted velocities and observed ones is defined as the $L_{2}$-norm of the misfit as

$$
\begin{aligned}
C\left(\alpha_{n}, c_{n}\right) & =\| V_{\bmod }\left(\alpha_{n}, c_{n}\right) \\
& -V_{\mathrm{obs}}\left(\alpha_{n}, c_{n}\right) \|^{2} .
\end{aligned}
$$

Within each inversion, the maximum iteration for loop on temperature is set to be 1500 , and at each temperature, all parameters have been perturbed 50 times according to equations 4 and 5 . Therefore, a total of 75,000 iterations in each VFSA inversion has been tested. 


\section{Sensitivity analysis of the inversion of crack distribution to clay}

Berea is not a clean quartz sandstone as is the Fontainebleau; it has some amount of clay. Because the clay elastic moduli can be substantially different from those of quartz, a small amount of clay can affect the elastic moduli of the composite and the ratio between the bulk and shear moduli. This ratio may result in different parameters of the inversion and especially may affect the aspect ratios of the small cracks. Therefore, it is worth spending some effort to investigate the impact of clay content on the effective elastic properties and inversion.

As shown in Figure 1, the effective elastic moduli $\langle K\rangle$ and $\langle\mu\rangle$ for a dry and water-saturated digitized rock sample with porosity of $19.2 \%$ vary with the concentration of clay and cracks. From this we can see that the magnitude of the decrease of elastic moduli due to increase of clay is much less than that due to increasing crack concentration. In these computations, we first include clay into the rock matrix occupied by pure quartz using the Voigt-Reuss-Hill average (VRH) (Mavko et al., 1998), then add cracks by using Kuster-Toksöz model. Even small increase in cracks can induce a significant amount of decrease in the effective elastic moduli, especially for cracks with smaller aspect ratios. Therefore, in this paper, we first put much effort into inverting crack distribution because any missing crack can impact the computation of the effective elastic property in a significant way.

In this section, we utilize the VFSA introduced in the previous section to find the best crack distribution. The laboratory data of Berea sandstone (BEREA 1) measured by Han (1986) for dry and water-saturated cases as listed in Table 2 are used as the observed data in the inversion. A total of 200 independent VFSA inversions were carried out for each case with different clay content. In Figure $6 \mathrm{a}$ and $6 \mathrm{c}$, we plot the mean and the standard deviation of the aspect ratios and concentrations of the nine sets of cracks changing with the clay content, and in Figure $6 \mathrm{~b}$ and $6 \mathrm{~d}$, we plot the very best inverted crack distributions in terms of aspect ratio and concentration out of the 200 best inversion results. From these figures, we can see that the variations of the aspect ratios and concentrations of cracks with clay content are generally small, which means the inversion results are not too sensitive to the clay content included in the matrix. From the inversion results shown in Figure 6, we can see that for Berea sandstones, cracks with aspect ratios of $10^{-2}$ are mostly left open at $40 \mathrm{MPa}$ and the concentration is in the order of $10^{-2}$. Our inverted results are consistent with those studied by Toksöz et al. (1976), who inverted the pore spectra of Boise sandstone and showed that at $50 \mathrm{MPa}$, cracks with aspect ratios of $10^{-2}$ were left open with the concentration in the order of $10^{-2}$ as well.
In Figure 7, we plot the variations of velocities of P- and S-waves for dry and water-saturated rocks with clay content by including the very best crack distribution inverted from the 200 independent VFSA inversions at each given clay content. From Table 2, we know the Berea sandstone (BEREA 1) Han (1986) used has porosity $19 \%$ and $6 \%$ clay, very similar to the sample we are using (Zhan et al., 2010). As we increase the clay content, the computed velocities, by including the cracks inverted, are getting closer to the laboratory data without greatly changing the inverted crack distributions. In Table 4, we compare the computed effective elastic properties of the digitized Berea sandstone with and without including cracks and clay. The values for rocks including cracks and clay are extracted from the data points shown in Figure 7. From this table, we can see that by adding cracks only into the rock matrix,
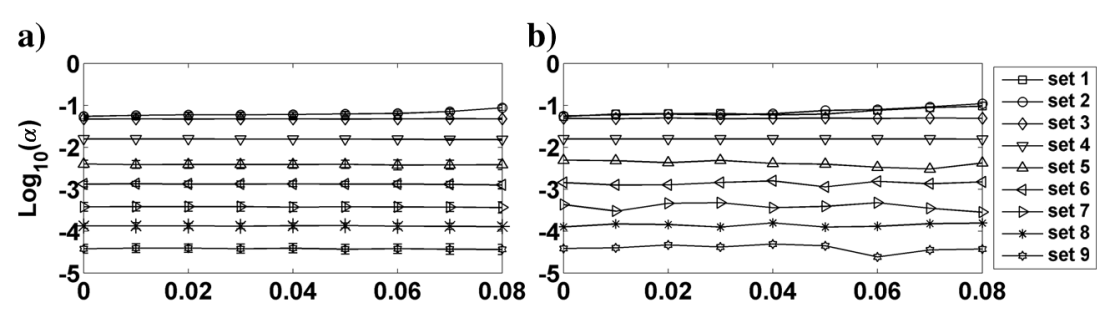

c)
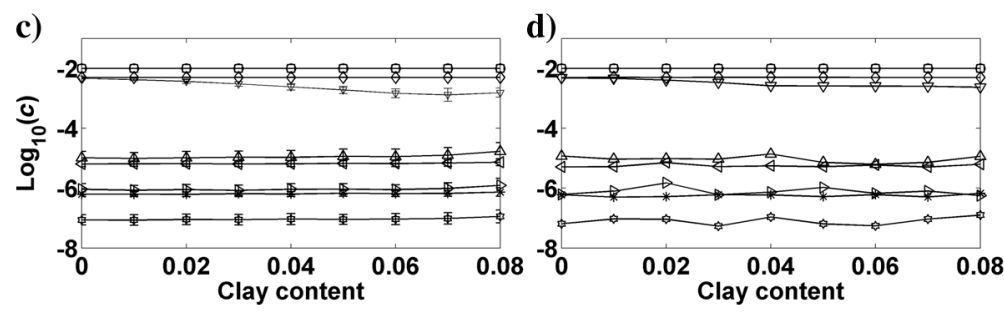

Figure 6. The inverted crack distribution - aspect ratio $(\alpha)$ and concentration $(c)$ - as a function of clay content after the VFSA inversion. The mean and standard deviation of the (a) aspect ratios and (c) concentrations of the nine sets of cracks, and the very best inverted (b) aspect ratios and (d) concentrations of the nine sets of cracks from the 200 independent VFSA inversions.
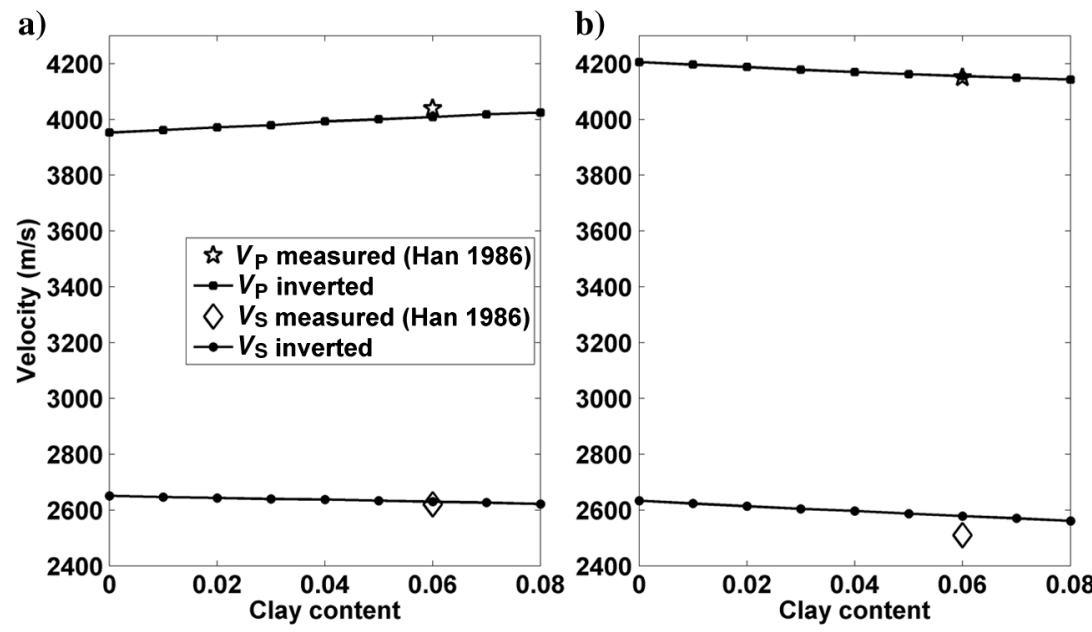

Figure 7. Comparison of the computed velocities (P-wave and S-wave) for dry and water-saturated rocks by including cracks, inverted at different clay content, to laboratory data of BEREA 1 measured by Han (1986). 
the computed effective velocities decrease by $16.2 \%$ for $V_{\mathrm{P}}$ and $13.9 \%$ for $V_{\mathrm{S}}$ for dry case, and by $9.3 \%$ for $V_{\mathrm{P}}$ and $11.3 \%$ for $V_{\mathrm{S}}$ for water-saturated case. By including clay into the matrix, the variations of velocities with respect to those without any clay are $-1.4 \%$ for $V_{\mathrm{P}}$ and $0.8 \%$ for $V_{\mathrm{S}}$ for dry case, and $1.2 \%$ for $V_{\mathrm{P}}$ and $2.3 \%$ for $V_{\mathrm{S}}$ for water-saturated case. From this comparison, we can conclude that the effect of clay on the effective elastic properties can be small enough to be ignored when compared to the effects due to the microcracks.

\section{ELASTIC PROPERTY PREDICTION OF BEREA SANDSTONE CONTAINING CRACKS AND CLAY}

After obtaining the crack distribution, we carry out computations on digitized rocks taking cracks into account. In this section, we first add inverted cracks into the host of the solid grain containing clay by using the Kuster-Toksöz model. Then we add and saturate the pore spaces resolved in the imaging process and compute the total effective elastic properties with the finite-element solver.

This sequence of adding inclusions - pores and cracks - is opposite to what we did in crack distribution inversion, where we first added and saturated the pore spaces to compute the intermediate effective elastic properties with the FEM solver, then inverted the crack distribution by using the VFSA method based on the Kuster-Toksöz model. According to the DEM model, the effective properties of composite, which depend on the sequence of adding different inclusions, generally could be different, but can be quite close in some cases.

\section{Prediction for dry and water-saturated Berea sandstone}

Based on the laboratory data of Berea sandstone (BEREA 1) measured by Han (1986) for dry and water saturation, we have inverted the crack distribution. Following the new computational sequence described, we can in turn predict the effective elastic properties of the digitized Berea sandstone for dry and water saturation as well.

As we have discussed with the data listed in Table 2, due to the small variations of the velocities among the different Berea sandstone, we can accept them all as the laboratory data for the digitized Berea sandstone we use in this paper. The average clay content for these Berea sandstone is about $7 \%$ and the average porosity is about $19.5 \%$; these are close to the digitized rock sample we use. To compute the effective elastic properties, we first need to compute the elastic moduli of the solid grain containing the cracks and clay. To do so, we first use the VRH average to include the $7 \%$ clay into pure quartz so as to update the rock matrix. Then adding the very best cracks inverted at $7 \%$ clay in the previous section into this updated rock matrix by using the Kuster-Toksöz model, we can obtain a new continuous rock matrix with both clay and cracks included.

Assigning the values of the effective elastic properties of the new rock matrix to solid grains of the $3 \mathrm{D}$ digitized Berea sandstone shown in light gray in Figure 3, we then can use the FEM solver to compute the final effective elastic properties for dry and water saturation. The final results are listed in Table 5 where we put the computed results side by side with laboratory measurements of Winkler (1985), Han (1986), and Best and McCann (1995) for comparison. The values, in percentage, below the laboratory data are the difference between computed velocities and corresponding laboratory measurements. We can see from these values that the predicted velocities match laboratory measurements quite well, and the differences fall within the error bounds observed among the laboratory data themselves. We inverted the distribution of cracks only with the laboratory measurements of BEREA 1 by Han (1986) for dry and water-saturated cases, the good match between computed results and the laboratory measurements of BEREA 1 should be expected. This also indicates that the sequence of adding inclusions defined by DEM model do lead to convergent results. The good match for other Berea sandstone indicates the capability of our method to predict the effective elastic properties of similar sandstone in terms of porosity and clay content.

\section{Prediction for brine- and oil-saturated Berea sandstone}

Instead of dry and water saturation, we can saturate the cracks and pores with brine and oil. Applying the same sequence as we did for dry and water-saturated cases, we can numerically predict the effective elastic properties of the digitized Berea sandstone for brine and oil saturation with the same crack distribution inverted from laboratory data for dry and water-saturated cases. This is similar to the idea of using Gassmann's model to do the fluid substitution (Gassmann, 1951) — predicting the effective elastic moduli of saturated sandstone with moduli of dry frame, or predicting the effective elastic moduli of rocks saturated with one fluid with the moduli of the same rocks when saturated with another fluid. However, our method not only captures the porosity but also the crack distributions and the complex microstructure of the pore space from X-ray CT image; this enables us to describe the rocks in more detail compared to the Gassmann's model. As listed in the Table 5, the predicted effective properties of brine and oil-saturated rocks also match the laboratory measurements by Winkler (1985) well. This again indicates the predicting power of the method when

Table 4. Comparison of the effects of the crack and clay content on the computed effective elastic properties of the digitized Berea sandstone.

Dry Water

\begin{tabular}{|c|c|c|c|c|c|c|c|c|c|}
\hline & & \\
\hline & & $V_{\mathrm{P}}(\mathrm{m} / \mathrm{s})$ & $\Delta v / v$ & $V_{\mathrm{S}}(\mathrm{m} / \mathrm{s})$ & $\Delta v / v$ & $V_{\mathrm{P}}(\mathrm{m} / \mathrm{s})$ & $\Delta v / v$ & $V_{\mathrm{S}}(\mathrm{m} / \mathrm{s})$ & $\overline{\Delta v / v}$ \\
\hline No crack & No clay & 4720 & & 3080 & & 5637 & & 2972 & \\
\hline With crack & No clay & 3953 & $-16.2 \%$ & 2651 & $-13.9 \%$ & 4205 & $-9.3 \%$ & 2634 & $-11.3 \%$ \\
\hline With crack & $6 \%$ clay & 4009 & $1.4 \%$ & 2630 & $-0.8 \%$ & 4155 & $-1.2 \%$ & 2578 & $-2.3 \%$ \\
\hline
\end{tabular}


Table 5. Comparison of computed effective properties of the 3D digitized Berea sandstone with laboratory data for different fluid saturations (unit: $\mathrm{m} / \mathrm{s}$ ).

Laboratory

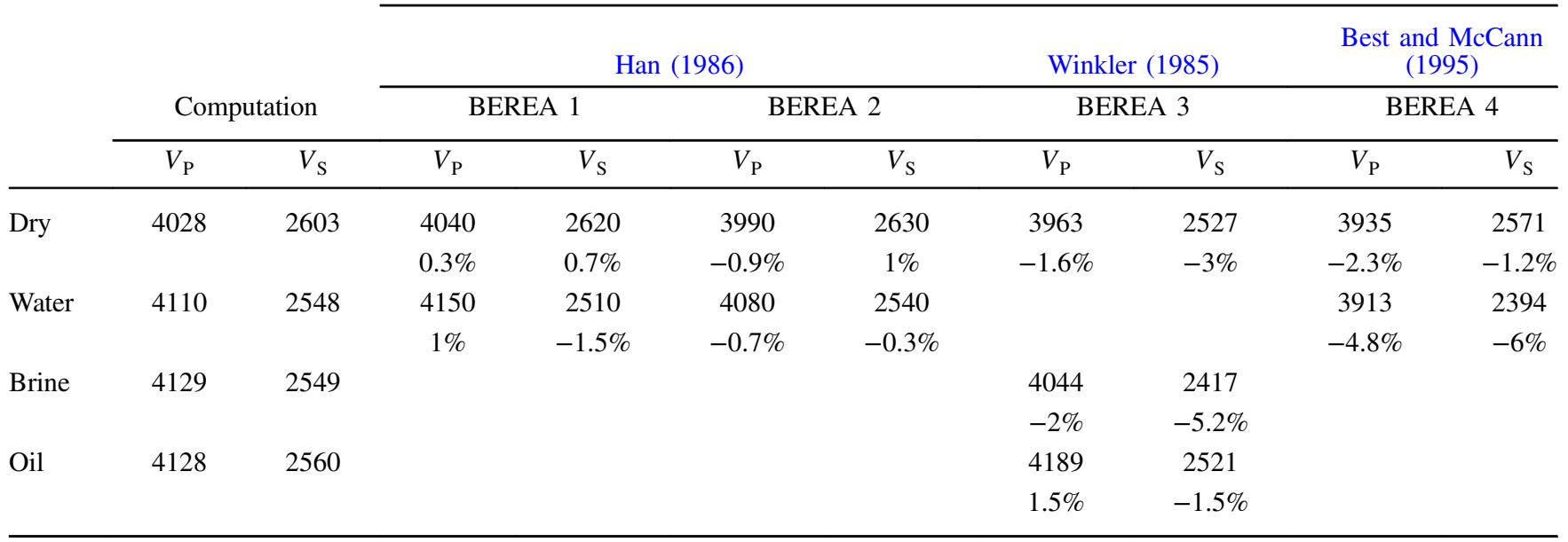

we apply it to the fluid substitution on the same or similar sandstone.

\section{DISCUSSION}

In this paper, we studied the effects of cracks lost in the imaging process on the computed effective elastic properties from 3D microtomographic images of Berea sandstone. By using the VRH average, we actually can take the clay into account. Ideally, we should identify one more solid phase, corresponding to clay in the segmented images so as to study not only the effect of clay content but also its spatial distribution on the computed effective elastic properties and the inversion of the crack distribution. In the computation, we then could assign elastic properties of clay onto the corresponding pixels in the segmented images so as to take clay into account. No matter what approach we take to consider the composition of minerals, cracks exist in all of them, in clay and between grains. The impact of cracks on elastic properties of rocks, therefore, is significant.

Because we use the Kuster-Toksöz model in the VFSA inversion, we assume random distribution of spheroidal cracks in rocks, though we know from the images that this is an approximation. A more detailed description of cracks can be obtained if highresolution images (at least in nanometer scale) were available. However, even if we had such high-resolution data, there would be the need to carry out some upscaling to make the computation feasible on $3 \mathrm{D}$ samples of realistic size. Combining effective media theory with the information about these small features obtained from imaging analysis is one way to accomplish this.

\section{CONCLUSION}

Realizing the significant impact of the small cracks lost during the imaging process on the computed effective elastic properties of digitized porous rocks, we developed a very fast simulated annealing inversion algorithm to recover the small cracks lost, in which we hybridized the numerical computation with effective media theories such as the differential effective medium theory and the KusterToksöz model. In the inversion, we only need a limited number of laboratory data of velocities of P- and S-waves, such as those measuring dry and water-saturated cases, to invert for crack distribution. Once the crack distribution is obtained, we are able to predict the effective elastic properties of rocks saturated with other types of fluids for the same or similar sandstone. The sensitivity analysis we carried out showed that crack distribution is not too sensitive to clay and clay only has a secondary effect on the computed effective elastic moduli compared to cracks. Because we used the elastic moduli computed from the digitized 3D rock as an intermediate step in our inversion, we actually captured most of the information about the microstructures of the rocks being imaged by X-ray CT scan. Therefore, the inversion results of cracks based on such digitized 3D rocks are more meaningful than those obtained solely by using a theoretical model, which has to invert the whole spectrum of crack distribution - both stiff pores and compliant cracks - rather than small cracks alone as in our inversion. With the crack distribution inverted from laboratory data for dry and water-saturated Berea sandstone BEREA 1, we could predict the effective elastic properties of other Berea sandstone for dry and for water, brine, and oil saturations.

\section{ACKNOWLEDGMENTS}

The authors wish to thank Schlumberger-Doll Research for funding the Canonical Rock Project and MIT ERL Founding Member Consortium for support on this project. During part of this work, Yang Zhang was supported as an ARAMCO Fellow.

\section{REFERENCES}

Arns, C., 2002, The influence of morphology on physical properties of reservoir rocks: Ph.D. thesis, University of New South Wales.

Arns, C., M. Knackstedt, W. Pinczewski, and E. Garboczi, 2002, Computation of linear elastic properties from microtomographic images: Methodology and agreement between theory and experiment: Geophysics, $\mathbf{6 7}$ 1396-1405, doi: 10.1190/1.1512785.

Arns, C., M. Madadi, A. Sheppard, and M. Knachstedt, 2007, Linear elastic properties of granular rocks derived from X-ray CT images: 77th Annual International Meeting, SEG Expanded Abstracts, 1711-1714. 
Berryman, J., 1992, Single-scattering approximations for coefficients in Biot equations of poroelasticity: Journal of the Acoustical Society of America, 91, 551-571, doi: 10.1121/1.402518.

Best, A., and C. McCann, 1995, Seismic attenuation and pore-fluid viscosity in clay-rich reservoir sandstone: Geophysics, 60, 1386-1397, doi: 10.1190/1.1443874.

Flannery, B., H. Deckman, W. Roberge, and K. Damico, 1987, 3-D X-ray microtomography: Science, 237, 1439-1444, doi: 10.1126/science.237 .4821 .1439 .

Fredrich, J., B. Menendez, and T. Wong, 1995, Imaging the pore structure of geomaterials: Science, 268, 276-279, doi: 10.1126/science.268 .5208 .276 .

Garboczi, E., 1998, Finite element and finite difference programs for computing the linear electric and linear elastic properties of digital images of random materials: Technical report, National Institute of Standards and Technology.

Garboczi, E., and A. Day, 1995, Algorithm for computing the effective linear elastic properties of heterogeneous materials - 3-D results for composites with equal phase Poisson ratios: Journal of the Mechanics and Physics of Solids, 43, 1349-1362, doi: 10.1016/0022-5096(95)00050-S.

Gassmann, F., 1951, Über die elastizität poröser medien: Vierteljahrsschrift der Naturforschenden Gesellschaft in Zurich, 96, 1-23.

Grechka, V., and M. Kachanov, 2006, Effective elasticity of rocks with closely spaced and intersecting cracks: Geophysics, 71, no. 3, D85-D91, doi: 10.1190/1.2197489.

Han, D., 1986, Effects of porosity and clay content on acoustic properties of sandstone and unconsolidated sediments: Ph.D. thesis, Stanford University.

Hudson, J., 1980, Overall properties of a cracked solid: Mathematical Proceedings of the Cambridge Philosophical Society, 88, 371-384, doi: $10.1017 / \mathrm{S} 0305004100057674$.

Ingber, L., 1989, Very fast simulated re-annealing: Mathematical and Computer Modeling, 12, 967-973, doi: 10.1016/0895-7177(89)90202-1.

Knackstedt, M., S. Latham, M. Madadi, A. Sheppard, T. Varslot, and C. Arns, 2009, Digital rock physics: 3D imaging of core material and correlations to acoustic and flow properties: The Leading Edge, 28, 28-33, doi: 10.1190/1.3064143.

Kuster, G., and M. Toksöz, 1974, Velocity and attenuation of seismic-waves in two-phase media: Part I. Theoretical formulation: Geophysics, 39 587-606, doi: 10.1190/1.1440450.

Mavko, G., T. Mukerji, and J. Dvorkin, 1998, The rock physics handbook: Cambridge University Press.

Murphy, W., K. Winkler, and R. Kleinberg, 1986, Acoustic relaxation in sedimentary rocks: Dependence on grain contacts and fluid saturation: Geophysics, 51, 757-766, doi: 10.1190/1.1442128.
O'Connell, R., and B. Budiansky, 1977, Viscoelastic properties of fluidsaturated cracked solids: Journal of Geophysical Research, 82, 5719-5735, doi: 10.1029/JB082i036p05719.

Roberts, A., and E. Garboczi, 2000, Elastic properties of model porous ceramics: Journal of the American Ceramic Society, 83, 3041-3048, doi: 10.1111/jace.2000.83.issue-12.

Saenger, E., O. Krueger, and S. Shapiro, 2006, Effective elastic properties of fractured rocks: Dynamic vs. static considerations: International Journal of Fracture, 139, 569-576, doi: 10.1007/s10704-006-0105-4.

Saenger, E., O. Kruger, and S. Shapiro, 2004, Numerical considerations of fluid effects on wave propagation: Influence of the tortuosity: Geophysical Research Letters, 31, L21613, doi: 10.1029/ 2004GL020970.

Saenger, E. H., 2008, Numerical methods to determine effective elastic properties: International Journal of Engineering Science, 46, 598-605, doi: 10.1016/j.ijengsci.2008.01.005.

Spanne, P., J. Thovert, C. Jacquin, W. Lindqust, K. Jones, and P. Adler, 1994, Synchrotron computed microtomography of porous-media Topology and transports: Physical Review Letters, 73, 2001-2004, doi: 10.1103/PhysRevLett.73.2001.

Toksöz, M., C. Cheng, and A. Timur, 1976, Velocities of seismic waves in porous rocks: Geophysics, 41, 621-645, doi: 10.1190/ 1.1440639.

Tran, D. T., C. S. Rai, and C. H. Sondergeld, 2008, Changes in crack aspectratio concentration from heat treatment: A comparison between velocity inversion and experimental data: Geophysics, 73, no. 4, E123-E132, doi: 10.1190/1.2928848.

Walsh, J., 1965, Effect of cracks on the compressibility of rock: Journal of Geophysical Research, 70, 381-389, doi: 10.1029/JZ070i002p00381.

Winkler, K., 1985, Dispersion analysis of velocity and attenuation in Berea sandstone: Journal of Geophysical Research, 90, 6793-6800, doi: 10.1029/JB090iB08p06793.

Wyllie, M., A. Gregory, and G. Gardner, 1958, An experimental investigation of factors affecting elastic wave velocities in porous media: Geophysics, 23, 459-493, doi: 10.1190/1.1438493.

Wyllie, M., A. Gregory, and L. Gardner, 1956, Elastic wave velocities in heterogeneous and porous media: Geophysics, 21, 41-70, doi: 10.1190/1.1438217.

Zhan, X., L. M. Schwartz, M. N. Toksöz, W. C. Smith, and F. D. Morgan, 2010, Pore-scale modeling of electrical and fluid transport in Berea sandstone: Geophysics, 75, no. 5, F135-142, doi: 10.1190/1.3463704.

Zhang, Y., 2010, Modeling of the effects of wave-induced fluid motion on seismic velocity and attenuation in porous rocks: Ph.D. thesis, Massachusetts Institute of Technology. 